\title{
Étude expérimentale de l'écoulement généré par un jet rond sur la surface d'un disque en rotation
}

\author{
KaCI BoussafeuR ${ }^{\mathrm{a}}$ \\ Laboratoire de mécanique, structure \& énergétique, Université Mouloud Mammeri de Tizi-ouzou, Algérie
}

Reçu le 10 février 2011, accepté le 19 juillet 2011

Résumé - On étudie expérimentalement l'écoulement turbulent pariétal produit par l'impact d'un jet frappant normalement un disque coaxial en rotation. La méthode utilisée est l'anémométrie à fil chaud : on mesure les vitesses moyennes, ainsi que les contraintes turbulentes dans un domaine où les effets de l'alimentation par le jet axial et ceux de la rotation du disque sont du même ordre de grandeur. On se place dans le cadre des approximations de couche limite pour obtenir une formulation simplifiée du problème. Par ailleurs, on met en évidence les paramètres sans dimensions qui jouent un rôle significatif. L'étude expérimentale est conduite en faisant varier ces paramètres. L'analyse des résultats expérimentaux fournit les échelles caractéristiques des vitesses et des longueurs, ainsi que le niveau de turbulence. De plus, elle donne des informations sur l'importance relative des différents termes des équations de Reynolds et permet de vérifier la validité de l'approximation de couche limite.

Mots clés : Écoulement / turbulent / pariétal / rotation / disque tournant / anémométrie à fil chaud

Abstract - Experimental study of the flow generated by a round jet on the surface of a disc in rotation. We study experimentally the swirling wall flow resulting from the impingement of a round jet on coaxial rotating disc. The method used is the hot wire anemometer: mean velocity and turbulent stresses were measured in the domain where the radial effects produced by the axial jet and the effects resulting from the rotation have the same order of magnitude. Boundary layer approximation is used in order to simplify the problem. Besides, one highlights the dimensionless parameters which have significant effects. The tests have been made for various values of these parameters. The analysis of our results provides the characteristics scales of velocity and length as well as the turbulence level. It brings some information about the relative importance of the terms standing in the Reynolds equations and gives access to a verification of the validity of the boundary layer approximation.

Key words: Flow / turbulent / parietal / rotation / rotating disc / hot wire anemometry

\section{Introduction}

Les écoulements tournants présentent à la fois un intérêt académique, scientifique et technologique. Sur le plan académique, on y simule des configurations inédites de turbulence. Des techniques de mesure sophistiquées permettent l'exploration exhaustive de l'écoulement. Par suite, des modèles capables d'approcher les valeurs expérimentales recueillies sont construits [1-5]. Sur le plan technologique, ces écoulements trouvent de nombreuses applications dans des domaines très variés comme l'astrophysique, la géophysique, l'informatique avec le refroidissement des disques durs, la météorologie, l'océanographie et surtout en turbomachines (compresseurs, turbines,

\footnotetext{
${ }^{a}$ Auteur pour correspondance : hamid261060@yahoo.fr
}

chambres de combustion, etc.). L'étude analytique de ces problèmes conduit souvent à des équations fort complexes et peu maniables. Le recours à l'expérimentation s'avère on ne peut plus nécessaire.

En 1921 Von Kármán étudie l'écoulement tournant induit par un disque en rotation dans un milieu immobile. Grâce à un changement de variables approprié, il a pu résoudre le problème en régime laminaire $[6,7]$. En écoulement turbulent, sa célèbre méthode intégrale permet, aussi, de trouver une solution approchée au problème. Cette solution a été améliorée numériquement en 1934 par Cochran. L'action d'un jet frappant normalement un disque immobile engendre sur le disque un jet pariétal purement radial. En adoptant une formule empirique, due à Blasius pour la zone de paroi, et en 


\section{Nomenclature}

\begin{tabular}{|lll|}
\hline$D$ & diamètre du tube & $\mathrm{m}$ \\
$H$ & distance axiale entre la sortie du jet et le disque & $\mathrm{m}$ \\
$N$ & vitesse de rotation du disque & $1 . \mathrm{s}^{-1}$ \\
$p$ & pression instantanée & $\mathrm{N} \cdot \mathrm{m}^{-2}$ \\
$\bar{p}$ & pression moyenne & $\mathrm{N} \cdot \mathrm{m}^{-2}$ \\
$p^{\prime}$ & pression fluctuante & $\mathrm{N}^{-2}$ \\
$p_{0}$ & pression atmosphérique & $\mathrm{N}^{-2}$ \\
$Q$ & débit volumique & $\mathrm{m}^{3} \cdot \mathrm{s}^{-1}$ \\
$R$ & rayon du disque & $\mathrm{m}$ \\
$R e$ & nombre de Reynolds & \\
$r, \theta, z$ & coordonnées cylindriques & \\
$S$ & nombre de rotation & $\mathrm{m} \cdot \mathrm{s}^{-1}$ \\
$u, v, w$ & composantes instantanées de la vitesse radiale, tangentielle et axiale & $\mathrm{m} \cdot \mathrm{s}^{-1}$ \\
$\bar{u}, \bar{v}, \bar{w}$ & composantes moyennes de la vitesse radiale, tangentielle et axiale & $\mathrm{m} \cdot \mathrm{s}^{-1}$ \\
$u^{\prime}, v^{\prime}, w^{\prime}$ & composantes fluctuantes de la vitesse radiale, tangentielle et axiale & $\mathrm{m} \cdot \mathrm{s}^{-1}$ \\
$\bar{u}_{\text {max }}$ & valeur maximale de $\bar{u}$ & $\mathrm{~m} \cdot \mathrm{s}^{-1}$ \\
$W_{0}$ & vitesse moyenne à la sortie du tube & $\mathrm{m}$ \\
$z$ & distance à partir de la surface du disque & \\
\hline & & $\mathrm{m}$ \\
\hline$\delta$ & épaisseur du jet : distance entre le disque mobile et le plan où $\bar{u}=\bar{u}_{\mathrm{max}} / 2$ & \\
$\varepsilon=D / H$ & paramètre de distorsion & $\mathrm{kg} \cdot \mathrm{m}^{-1} \cdot \mathrm{s}^{-2}$ \\
$\zeta$ & contrainte de cisaillement & $\mathrm{kg} \cdot \mathrm{m}^{-1} \cdot \mathrm{s}^{-1}$ \\
$\mu$ & viscosité dynamique & $\mathrm{kg} \cdot \mathrm{m}^{-3}$ \\
$\rho$ & masse volumique & $\mathrm{m}{ }^{2} \cdot \mathrm{s}$ \\
$v$ & viscosité cinématique & \\
$\Omega$ & taux de rotation $\Omega=2 \pi N$ & \\
\hline & & \\
\hline
\end{tabular}

appliquant un modèle de viscosité turbulente pour la zone extérieure, Glauert trouva deux solutions qui se raccordent aux points où la vitesse moyenne est maximale et la contrainte tangentielle nulle $[8,9]$.

Le problème du jet pariétal sur un disque lorsque les vitesses radiales et celles induites par la rotation du disque sont du même ordre de grandeur, traité dans la référence [10], objet de notre étude, a pour limites les deux écoulements sus cités.

La figure 1 illustre l'installation. Un jet d'air incompressible sort d'un tube circulaire de diamètre $D$ pour venir percuter perpendiculairement un disque lisse, en rotation, de rayon $R$, de vitesse $N$. Loin du centre du disque, dans la direction radiale, on a une région, notée II sur la figure, objet de cette étude, où les effets du jet radial et de la rotation du disque sont équivalents. La distance axiale entre la surface du disque et la sortie du jet est notée $H$.

\section{Formulation mathématique du problème}

Étant donné les propriétés de symétrie du problème, il est indiqué de formuler les équations régissant le problème en coordonnées cylindriques :

$$
\frac{1}{r} \frac{\partial}{\partial r}(r u)+\frac{1}{r} \frac{\partial v}{\partial \theta}+\frac{\partial w}{\partial z}=0
$$

$$
\begin{gathered}
\rho\left(\frac{\mathrm{d} u}{\mathrm{~d} t}-\frac{v^{2}}{r}\right)=F_{r}-\frac{\partial p}{\partial r}+\mu\left(\Delta u-\frac{u}{r^{2}}-\frac{2}{r^{2}} \frac{\partial v}{\partial \theta}\right) \\
\rho\left(\frac{\mathrm{d} v}{\mathrm{~d} t}+\frac{u v}{r}\right)=F_{\theta}-\frac{1}{r} \frac{\partial p}{\partial \theta}+\mu\left(\Delta v-\frac{v}{r^{2}}+\frac{2}{r^{2}} \frac{\partial u}{\partial \theta}\right) \\
\rho \frac{\mathrm{d} w}{\mathrm{~d} t}=F_{z}-\frac{\partial p}{\partial z}+\mu \Delta w
\end{gathered}
$$

avec :

$$
\begin{aligned}
& \frac{\mathrm{d}}{\mathrm{d} t}=\frac{\partial}{\partial t}+u \frac{\partial}{\partial r}+\frac{v}{r} \frac{\partial}{\partial \theta}+w \frac{\partial}{\partial z}, \\
& \Delta=\frac{\partial^{2}}{\partial r^{2}}+\frac{1}{r} \frac{\partial}{\partial r}+\frac{1}{r^{2}} \frac{\partial^{2}}{\partial \theta^{2}}+\frac{\partial^{2}}{\partial z^{2}}
\end{aligned}
$$

$F_{r}, F_{\theta}, F_{Z}$ sont les composantes du champ des forces extérieures suivant $r, \theta, z$.

Il est impossible de résoudre ces équations par voie analytique, a fortiori, en mouvement turbulent. Dans ce dernier cas, on aborde classiquement le problème par l'approche de Reynolds, consistant à décomposer toute grandeur physique $g$ en une partie moyenne $\bar{g}$, indépendante du temps en écoulement stationnaire et une grandeur fluctuante $g^{\prime}$, de moyenne temporelle nulle : $g=\bar{g}+g^{\prime}$. On obtient les équations, dites de Reynolds, après avoir pris la moyenne et compte tenu des hypothèses suivantes :

- Écoulement permanent, 


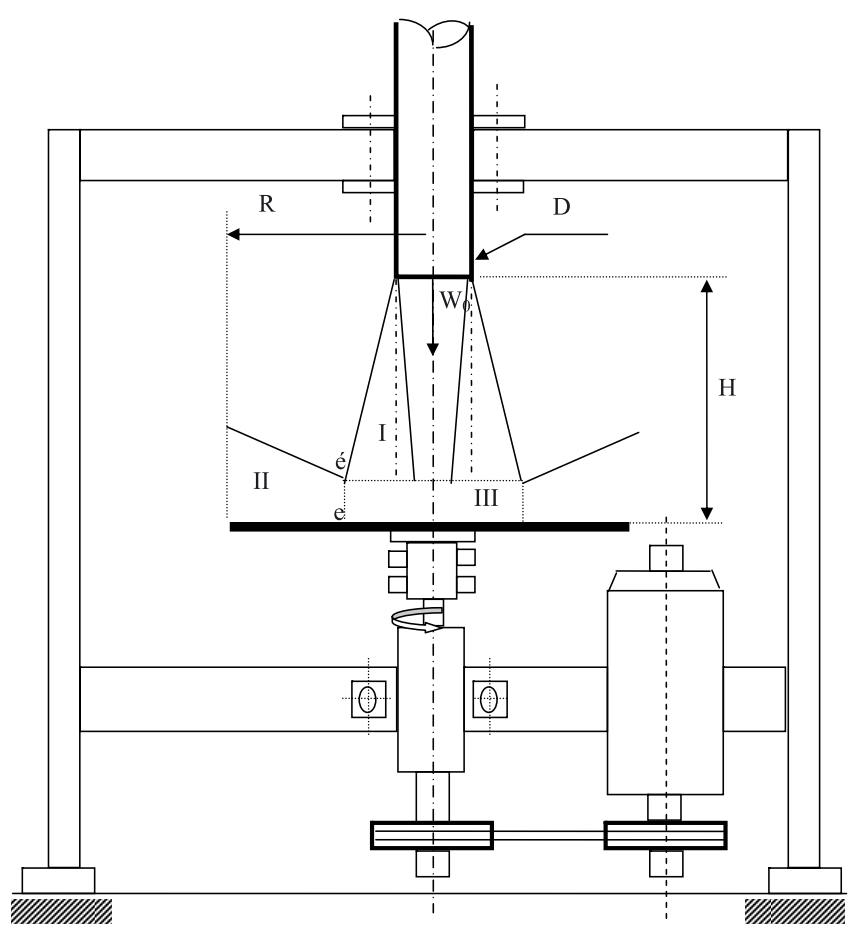

Fig. 1. Installation.

- Écoulement axisymétrique,

- Forces extérieures négligeables.

$$
\begin{gathered}
\frac{1}{r} \frac{\partial}{\partial r}(r \bar{u})+\frac{\partial \bar{w}}{\partial z}=0 \\
\bar{u} \frac{\partial \bar{u}}{\partial r}+\bar{w} \frac{\partial \bar{u}}{\partial z}-\frac{\bar{v}^{2}}{r}=-\frac{1}{\rho} \frac{\partial \bar{p}}{\partial r}+v\left(\frac{\partial}{\partial r}\left(\frac{1}{r} \frac{\partial}{\partial r}(r \bar{u})\right)+\frac{\partial^{2} \bar{u}}{\partial z^{2}}\right) \\
-\frac{1}{r} \frac{\partial}{\partial r} r \overline{u^{\prime 2}}+\frac{\overline{v^{\prime 2}}}{r}-\frac{\partial \overline{u^{\prime} w^{\prime}}}{\partial z} \\
\bar{u} \frac{\partial \bar{v}}{\partial r}+\bar{w} \frac{\partial \bar{v}}{\partial z}+\frac{\overline{u v}}{r}=v\left(\frac{\partial}{\partial r}\left(\frac{1}{r} \frac{\partial}{\partial r}(r \bar{v})\right)+\frac{\partial^{2} \bar{v}}{\partial z^{2}}\right) \\
-\frac{\partial}{\partial r} \frac{\partial u^{\prime} v^{\prime}}{2}-2 \frac{\overline{u^{\prime} v^{\prime}}}{r}-\frac{\partial \overline{v^{\prime} w^{\prime}}}{\partial z} \\
\left.\bar{u} \frac{\partial}{\partial r}+\bar{w} \frac{\partial \bar{w}}{\partial z}=-\frac{\partial \bar{p}}{\rho} \frac{(7)}{\partial z}\left(\frac{1}{r} \frac{\partial^{2}}{\partial r^{2}}(r \bar{w})\right)+\frac{\partial^{2} \bar{w}}{\partial z^{2}}\right) \\
-\frac{\partial}{\partial z} \frac{1}{w^{\prime 2}}-\frac{1}{r} \frac{\partial r \overline{u^{\prime} w^{\prime}}}{\partial r}
\end{gathered}
$$

Les conditions aux limites sont :

1. Adhérence sur le disque solide :

$$
\bar{u}=\bar{w}=u^{\prime}=v^{\prime}=w^{\prime}=0 ; \bar{v}=\Omega r \text { pour } z=0, r<R
$$

2. Adhérence sur le tube :

$$
\bar{u}=\bar{v}=\bar{w}=u^{\prime}=v^{\prime}=w^{\prime}=0 \operatorname{pour} r=D / 2 ; z>H
$$

3. À la sortie du tube :

$$
\begin{aligned}
\bar{u}=\bar{v} & =\bar{w}=u^{\prime}=v^{\prime}=w^{\prime}=0 ; \\
\bar{w} & =-W_{0} \text { et } \bar{p}=p_{0} \text { pour } z=H, r \leq D / 2
\end{aligned}
$$

Si l'on se rapporte aux équations de Navier Stokes (1), (2), (3) et (4), en tenant compte des hypothèses caractéristiques du problème énoncé précédemment, on constate que l'on dispose d'un système fermé de quatre équations à quatre inconnues $u, v, w, p$. Le fait de décomposer les grandeurs instantanées en valeurs moyennes et fluctuantes conduit aux équations (5), (6), (7) et (8) qui sont toujours au nombre de quatre, mais introduit six inconnues supplémentaires qui sont les composantes du tenseur des contraintes turbulentes. Pour les fermer, on peut écrire les équations de transport de la turbulence. Malheureusement, ces équations font intervenir d'autres inconnues, telles que les corrélations triples de vitesses et les corrélations vitesses pression.

Pour réduire le nombre d'inconnues et donc simplifier le problème, on utilise les approximations de couche limite. La méthode consiste à introduire pour chaque grandeur une grandeur de référence de même nature et telle que la grandeur normalisée correspondante, égale au rapport de la grandeur physique à la grandeur de référence, soit d'ordre 1. Dans les équations écrites sous forme normalisée apparaissent les quantités sans dimensions indépendantes des inconnues, qu'on appelle les paramètres d'approximations. Lorsque ces paramètres prennent des valeurs limites, on peut obtenir une simplification des équations de Reynolds.

Écrivons, donc, ces équations sous forme normalisée en introduisant ces paramètres réduits :

$$
\begin{aligned}
\hat{r}=\frac{r}{R} ; \quad \hat{z} & =\frac{z}{\varepsilon R} ; \quad \hat{\bar{u}}=\frac{\bar{u}}{W_{0}} ; \hat{\bar{v}}=\frac{\bar{v}}{\Omega R} ; \\
\hat{\bar{w}} & =\frac{\bar{w}}{W_{\mathrm{n}}} ; \quad \overline{u_{i}{ }^{\prime} u_{j}{ }^{\prime}}=\frac{\overline{u_{i}{ }^{\prime} u_{j}^{\prime}}}{\lambda W_{0}^{2}} ; \quad \hat{\bar{p}}=\frac{\bar{p}}{E \rho W_{0}^{2}}
\end{aligned}
$$

$W_{\mathrm{n}}, \lambda$ et $E$ sont des constantes telles que $\hat{\bar{w}}, \overline{u_{i}^{\prime} u_{j}^{\prime}}$ et $\hat{\bar{p}}$ soient d'ordre 1.

Portons (12) dans (5). Pour que l'équation de continuité n'ait pas de solution triviale il faut que : $W_{\mathrm{n}}=\varepsilon W_{0}$. On obtient alors :

$$
\frac{1}{\hat{r}} \frac{\partial \hat{r} \hat{\bar{u}}}{\partial \hat{r}}+\frac{\partial \hat{\bar{w}}}{\partial \hat{z}}=0
$$

$$
\begin{aligned}
& \hat{\bar{u}} \frac{\partial \hat{\bar{u}}}{\partial \hat{r}}+\hat{\bar{w}} \frac{\partial \hat{\bar{u}}}{\partial \hat{z}}-S^{2} \frac{\hat{\hat{v}}^{2}}{\hat{r}}=-E \frac{\partial \hat{\bar{p}}}{\partial \hat{r}}+\frac{S}{R_{e}} \frac{\partial}{\partial \hat{r}}\left(\frac{1}{\hat{r}} \frac{\partial}{\partial \hat{r}}(r \hat{\bar{u}})\right) \\
& +\frac{S}{\varepsilon^{2} R_{e}} \frac{\partial^{2} \hat{\bar{u}}}{\partial \hat{z}^{2}}-\frac{\lambda}{\hat{r}} \frac{\partial}{\partial \hat{r}}\left(\hat{r} \hat{u^{\prime 2}}\right)+\frac{\lambda \hat{v^{\prime 2}}}{\hat{r}}-\frac{\lambda}{\varepsilon} \frac{\partial \hat{u^{\prime} w^{\prime}}}{\partial \hat{z}} \\
& S\left(\hat{\bar{u}} \frac{\partial \hat{\bar{v}}}{\partial \hat{r}}+\hat{\bar{w}} \frac{\partial \hat{\bar{v}}}{\partial \hat{z}}+\frac{\hat{u} \hat{v}}{\hat{r}}\right)=\frac{S^{2}}{R_{e}} \frac{\partial}{\partial \hat{r}}\left(\frac{1}{\hat{r}} \frac{\partial}{\partial \hat{r}}(\hat{r} \hat{\bar{v}})\right) \\
& +\frac{S^{2}}{\varepsilon^{2} R_{e}} \frac{\partial^{2} \hat{\bar{v}}}{\partial \hat{z}^{2}}-\lambda \frac{\partial\left(\hat{u^{\prime} v^{\prime}}\right)}{\partial \hat{r}}-2 \lambda \frac{\hat{u^{\prime} v^{\prime}}}{\hat{r}}-\frac{\lambda}{\varepsilon} \frac{\partial \hat{v^{\prime} w^{\prime}}}{\partial \hat{z}}
\end{aligned}
$$




$$
\begin{aligned}
\hat{\bar{u}} \frac{\partial \hat{\bar{w}}}{\partial \hat{r}}+\hat{\bar{w}} \frac{\partial \hat{\bar{w}}}{\partial \hat{z}} & =-\frac{E}{\varepsilon^{2}} \frac{\partial \hat{\bar{p}}}{\partial \hat{z}}+\frac{S}{R_{e}} \frac{1}{\hat{r}} \frac{\partial^{2}(\hat{r} \hat{\bar{w}})}{\partial \hat{r}^{2}} \\
& -\frac{\lambda}{\varepsilon^{2}} \frac{\partial \hat{w^{\prime 2}}}{\partial \hat{z}}+\frac{S}{\varepsilon^{2} R_{e}} \frac{\partial^{2} \hat{w}}{\partial \hat{z}^{2}}-\frac{\lambda}{\hat{r}} \frac{\partial}{\partial \hat{r}}\left(\hat{r} \hat{u^{\prime} w^{\prime}}\right)
\end{aligned}
$$

avec $S=\frac{\Omega R}{W_{0}}$ et $R_{e}=\frac{\Omega R^{2}}{v}$.

Notre étude se fait dans la zone où les effets de l'alimentation par le jet axial et ceux de la rotation du disque sont équivalents, ce qui donne $S$ d'ordre 1 . Les cas $S \gg 1$, $S \ll 1$ ont déjà été étudiés : ils correspondent respectivement au problème d'un disque en rotation sans alimentation radiale [11-16] et au problème du jet purement radial $[8,9]$. Compte tenu, donc, de $S$ proche de 1 et de $\lambda=\varepsilon$, on obtient en négligeant tous les termes petits au regard d'autres retenus et en revenant aux grandeurs physiques :

$$
\begin{gathered}
\frac{\partial}{\partial r}(r \bar{u})+r \frac{\partial \bar{w}}{\partial z}=0 \\
\bar{u} \frac{\partial \bar{u}}{\partial r}+\bar{w} \frac{\partial \bar{u}}{\partial z}-\frac{\bar{v}^{2}}{r}=\frac{\partial}{\partial z}\left(\frac{\tau_{r z}}{\rho}-\overline{u^{\prime} w^{\prime}}\right) \\
\bar{u} \frac{\partial \bar{v}}{\partial r}+\bar{w} \frac{\partial \bar{v}}{\partial z}+\frac{\overline{u v}}{r}=\frac{\partial}{\partial z}\left(\frac{\tau_{\theta z}}{\rho}-\overline{v^{\prime} w^{\prime}}\right) \\
\tau_{r z}=\mu \partial \bar{u} / \partial z \quad \tau_{\theta z}=\mu \partial \bar{v} / \partial z
\end{gathered}
$$

Conditions aux limites :

$$
\begin{aligned}
& \bar{u}=\bar{w}=u^{\prime}=v^{\prime}=w^{\prime}=0 ; \quad \bar{v}=\Omega r \text { pour } z=0 \\
& \bar{u}=\bar{v}=u^{\prime}=w^{\prime}=0=\frac{\partial \bar{u}}{\partial z}=\frac{\partial \bar{v}}{\partial z}=0 ; \text { pour } z=\Delta
\end{aligned}
$$

$\Delta$ étant l'épaisseur du jet.

\section{3 Étude expérimentale}

Le dispositif expérimental comprend essentiellement une installation principale et des appareils de mesure et d'acquisition. On dispose, également, d'appareils secondaires, notamment un compresseur, deux réservoirs, un filtre à air et une armoire de climatisation. L'air en surpression, fourni par le compresseur, passe à travers un filtre métallique qui le débarrasse de ses impuretés et va à deux réservoirs. Il est ensuite acheminé vers l'installation à travers une conduite de section circulaire équipée d'un régulateur de débit. L'armoire de climatisation se trouve dans la salle où sont effectuées les expériences. Elle sert à maintenir constante la température ambiante.

L'installation principale est constituée par un disque en aluminium de diamètre $75 \mathrm{~cm}$ et d'épaisseur $2 \mathrm{~cm}$, solidaire d'un palier et qui peut être entraîné à l'aide d'un moteur à courant continu de puissance $2 \mathrm{~kW}$, équipé d'un variateur de vitesse et d'un système de poulies. Sa vitesse peut varier entre 0 et 4000 tr. $\mathrm{min}^{-1}$; elle est mesurée à l'aide d'un capteur électromagnétique à affichage numérique direct de précision 3 tr. $\min ^{-1}$, contrôlée à l'aide d'un stroboscope. Le réglage de l'alimentation se
Tableau 1. Données du problème : $H / D=6,25,2,5$.

\begin{tabular}{ccccc}
\hline & \multicolumn{3}{c}{$R e^{*} 10^{-6}$} \\
\cline { 2 - 5 } & 0,894 & 1,115 & 1,336 & \\
\hline $2 \pi \mathrm{NR}\left(\mathrm{m}_{\mathrm{s}} \mathrm{s}^{-1}\right) \rightarrow$ & 39,25 & 49,06 & 58,87 & \\
$W_{0}=\frac{4 Q}{\pi D^{2}}\left(\mathrm{~m} \cdot \mathrm{s}^{-1}\right) \downarrow$ & & & & \\
22,12 & 1,77 & 2,22 & 2,66 & $S$ \\
33,17 & 1,18 & 1,48 & 1,77 & \\
44,23 & 0,89 & 1,11 & 1,33 & \\
\hline
\end{tabular}

fait par une micro vanne de contrôle; le débit est mesuré à l'aide d'un rotamètre dont la précision est d'environ $2 \%$.

L'ensemble des appareils est fixé solidement sur une structure rigide et lourde afin d'éviter les vibrations. À une distance $H$ de la surface du disque se trouve un tube circulaire de diamètre intérieur $D$, fixé solidement à la même structure que l'ensemble des appareils décrits précédemment. Un jet issu du tube vient frapper perpendiculairement le disque en rotation. Le tube peut coulisser verticalement par simple desserrage des vis qui le retiennent à la structure rigide.

On a utilisé l'anémométrie à fil chaud pour étudier le problème. Par conséquent la vitesse moyenne et les fluctuations sont mesurées à l'aide de sondes à fils chauds simples et croisés de $1,2 \mathrm{~mm}$ de longueur et de $5 \mu \mathrm{m}$ de diamètre (Platinum Plated tungsten). La précision est de l'ordre de 1,5\%. L'acquisition et le stockage des paramètres se font simultanément. Le programme de traitement calcule les valeurs moyennes des vitesses et le tenseur de Reynolds. L'étalonnage de la sonde s'effectue dans le noyau d'un jet libre circulaire. La sonde et un tube de Pitot sont placés dans une même section pour avoir la même vitesse. La sonde est reliée à l'anémomètre à température constante dont la tension est affichée digitalement sur le module. Pour avoir une bonne courbe d'étalonnage, il suffit de faire varier la vitesse de sortie du jet et d'exécuter plusieurs essais. On détermine, ensuite, les constantes de la fonction de transfert du linéarisateur, qu'on introduit dans le module.

La conduite des essais nécessite la définition des paramètres réduits. On les retrouve par l'analyse dimensionnelle, par un choix judicieux des grandeurs physiques du problème. Le théorème de Vaschy Buckingham permet d'écrire :

$$
\frac{G i}{\bar{u}_{\max }}=f_{i}^{\prime}\left(S, R e, \frac{H}{D}, \frac{r}{D}, \frac{z}{\delta}\right)
$$

$S=\frac{2 \pi N R}{W_{0}}=\frac{\pi^{2} N R D^{2}}{2 Q}$ est le nombre de rotation et $R e=\frac{2 \pi N R^{2}}{v}$ le nombre de Reynolds. L'introduction du rayon du disque est faite pour retrouver le même nombre adimensionnel que celui utilisé à la section 2. Le nombre $S$ exprime le rapport entre deux vitesses : azimutale et débitante.

Le tableau 1 représente les données du problème. Pour un diamètre $D=0,04 \mathrm{~m}$ on a pris deux valeurs de la hauteur du jet $H(0,25 \mathrm{~m}$ et $0,1 \mathrm{~m})$. On a, donc, deux valeurs 
de $H / D$. Pour une valeur de $H / D$ on a considéré trois débits, auxquels correspondent neuf vitesses de rotation du disque, ce qui donne neuf valeurs à $S$ pour chaque valeur de $H / D$.

Les mesures ont été effectuées à différentes positions radiales : $r / D=3,375,3,750,4,125,4,875,5,625,6,375$, 7,375 .

\section{Analyse des résultats}

Les résultats expérimentaux sont représentés sous forme adimensionnelle pour différentes valeurs de $H / D$, à diverses positions radiales $r / D$, en prenant plusieurs valeurs de $S$ et de $R e$. Cette représentation permet en particulier de tester la validité de l'existence d'une solution de similitude.

\subsection{Grandeurs moyennes}

\subsubsection{Vitesse radiale moyenne}

Les profils de $\bar{u} / \bar{u}_{\max }$ présentent presque une similitude lorsque $r / D$ est faible (Fig. 2a). Ils avoisinent le cas du jet purement radial, sans rotation du disque. Pour $r / D$ grand $\bar{u} / \bar{u}_{\max }$ dépend de $S$. Les profils s'éloignent de la solution de Glauert [8], correspondant au jet radial sur un disque sans rotation, pour des grandes positions radiales et/ou pour les grandes valeurs de $S$.

On peut, aussi, remarquer que le lieu de la vitesse maximale s'approche de la surface du disque lorsque $r / D$ et $S$ augmentent séparément ou ensemble (Fig. 2b). À titre de comparaison, la distance adimensionnelle entre le disque et les points où $\bar{u}=\bar{u}_{\max }$ est égale à 0,23 pour le jet purement radial et à 0,19 pour l'écoulement de Von Kármán $[17,18]$.

Pour la même position radiale réduite et aux mêmes valeurs de $S$ et de $R e$, on constate une faible différence dans le profil des vitesses. Il faudrait vraisemblablement une variation importante de $H / D$ pour qu'une différence sensible soit perceptible. Quand $H / D$ tend vers l'infini, $\bar{u} / \bar{u}_{\max }$ doit s'approcher du profil correspondant à un disque en rotation sans alimentation.

\subsubsection{Vitesse tangentielle moyenne}

On a porté sur les figures $3 \mathrm{a}$ et $3 \mathrm{~b}$ le rapport $\bar{v} / \Omega r$ en fonction de $z / \delta . \bar{v} / \Omega r$ ne dépend pas séparément de $S$ et de $R e$ mais du rapport $\frac{S}{R_{e}}=\frac{\pi D^{2} v}{4 R Q}$, ce qui signifie que la rotation du disque n'affecte pas ce profil des vitesses : plus ce rapport est petit, plus $\bar{v} / \Omega r$ est grand (Fig. 3a).

L'influence du rapport $r / D$ est significative puisque $\bar{v} / \Omega r$ diminue quand il augmente (Fig. 3b). Pour les grandes distances radiales et/ou les grandes valeurs de $\frac{S}{R_{e}}=\frac{\pi D^{2} v}{4 R Q}$, on obtient des courbes dont l'allure est similaire à celle d'un disque en rotation sans alimentation radiale; courbe en continu, due à Cham et Head de la figure $3 \mathrm{a}$.

Le paramètre $H / D$ a une légère influence sur les profils $\bar{v} / \bar{v}_{\max }$. De la même manière que pour $\bar{u} / \bar{u}_{\max }$, il faudrait des grandes variations de $H / D$ pour que la différence puisse être visible. Cela découle du fait que pour $H / D$ élevé, $\bar{v} / \Omega r$ doit coïncider avec le profil de Von Kármán.

\subsubsection{Rapport des vitesses moyennes radiales et tangentielles}

Les profils de $\bar{u} / \bar{v}$ en fonction de $z / \delta$ sont représentés sur les figures $4 \mathrm{a}$ et b pour deux positions radiales et une valeur de $H / D$ : plus $r / D$ est grand, plus l'influence de $R e$ et $S$ est importante. Sur la figure $4 \mathrm{~b}$, on a fait varier $r / D$ et on a maintenu constants $S$ et $R e$. On remarque que $\bar{u} / \bar{v}$ varie en sens inverse de $r / D$.

On peut distinguer trois zones suivant la valeur de $z$ :

- Dans la sous-couche visqueuse la vitesse radiale est négligeable devant la vitesse tangentielle.

- Très loin de la surface du disque $\bar{u} / \bar{v}$ est de l'ordre de 1.

- Dans la zone intermédiaire il est difficile de comparer $\bar{u}$ à $\bar{v}$ du fait que l'influence des paramètres $S$ et $R e$ y est sensible, notamment pour les valeurs élevées de $r / D$. Pour les faibles valeurs de $r / D, \bar{u}$ est le produit de $\bar{v}$ par une fonction de $z / \delta$.

Si les trois zones pouvaient être délimitées, il serait possible d'écrire les équations (17), (18), (19) sous une forme plus simple pour les faibles valeurs de $r / D$.

\subsection{Contraintes turbulentes}

En ce qui concerne les contraintes turbulentes on peut en gros distinguer deux zones : une région interne, proche de la surface du disque et une région externe. Dans la première région les paramètres sans dimensions définis précédemment ont un effet important et l'épaisseur de cette région en dépend d'ailleurs, de même que de $r / D$. D'après l'ensemble de nos résultats expérimentaux, on peut avancer que l'épaisseur de ce domaine n'excède pas $\delta$. Dans la deuxième région on a approximativement une similitude ; la rotation $N$, le diamètre $D$ n'y ont pas d'effets conséquents sur les profils des contraintes turbulentes.

\subsubsection{Contrainte turbulente radiale}

Les figures $5 \mathrm{a}$ et $\mathrm{b}$ représentent $\frac{\overline{u^{\prime 2}}}{\overline{u_{\max }^{2}}}$ en fonction de $z / \delta$ pour différentes valeurs de $S, R e$ et à $H / D, r / D$ fixés. Dans la zone externe, il y a presque similitude car tous les profils se superposent. Dans la zone interne, proche de la paroi, quand $S$ ou $R e$ diminuent, l'intensité de la turbulence augmente d'autant plus que $r / D$ est grand (Figs. 5a et b). 


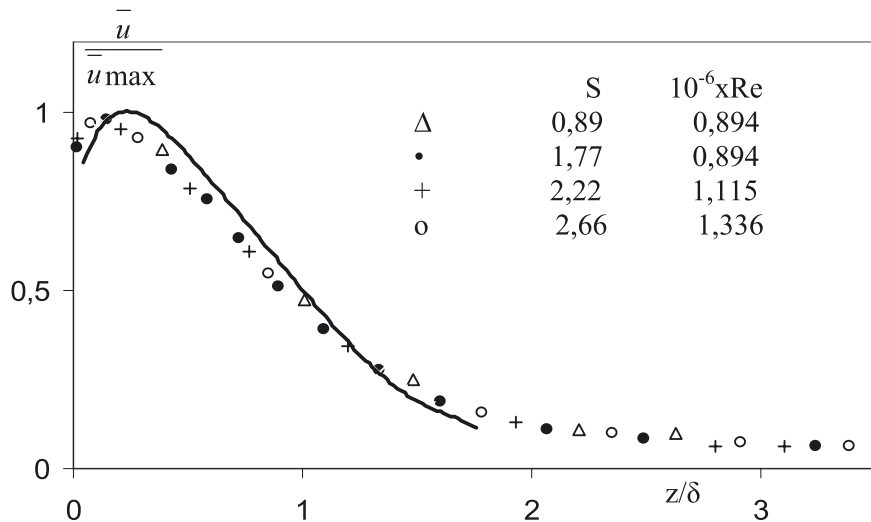

(a)

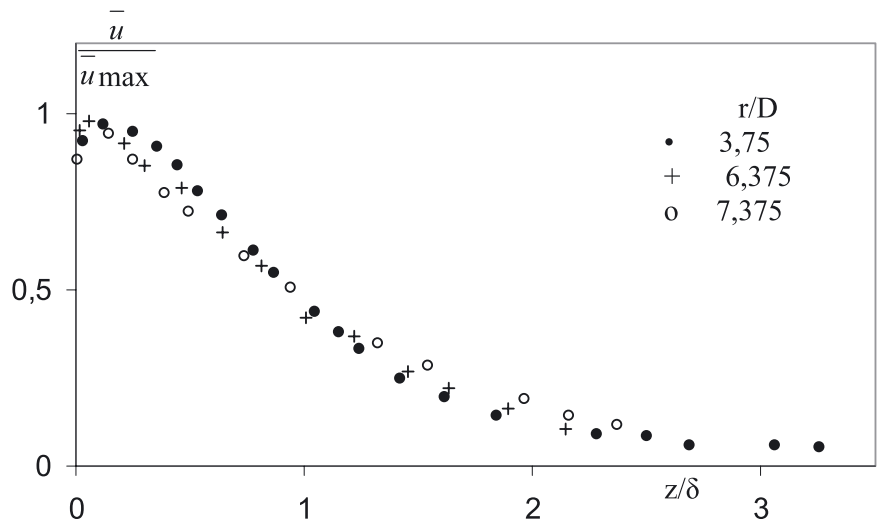

(b)

Fig. 2. (a) Vitesses radiales réduites en fonction de $z / \delta . H / D=2,5, r / D=3,75$. (b) Vitesses radiales réduites en fonction de $z / \delta . H / D=6,25, S=1,77, R e=0,894 \times 10^{6}$.

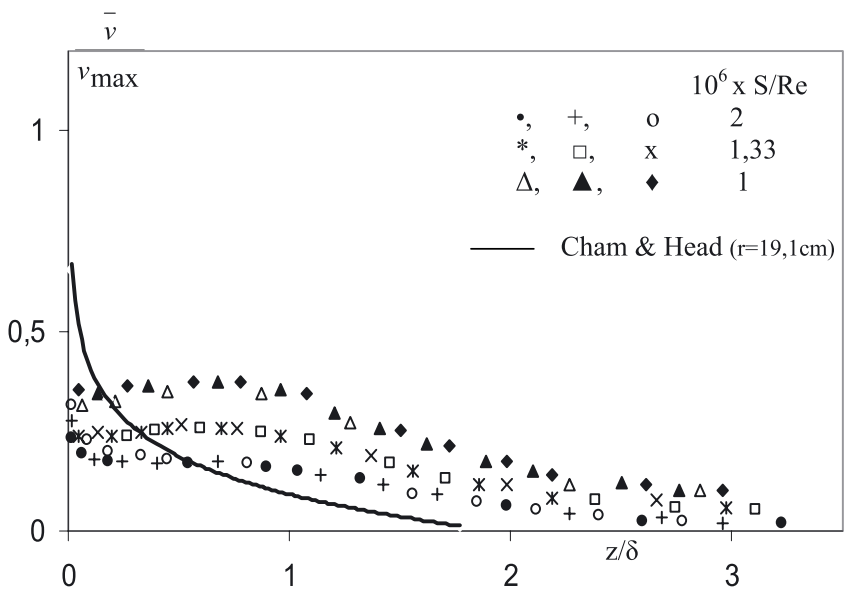

(a)

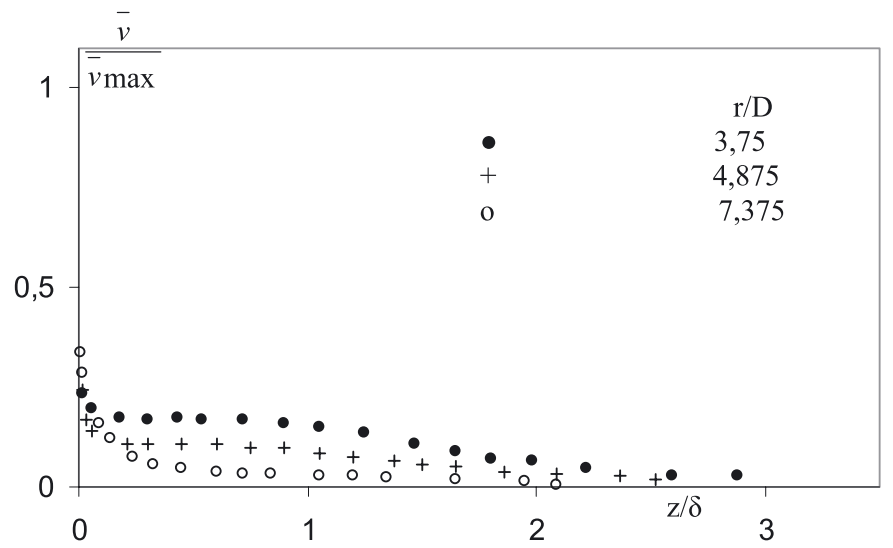

(b)

Fig. 3. (a) Vitesses tangentielles réduites en fonction de $z / \delta . H / D=6,25, r / D=3,75$. (b) Vitesses tangentielles réduites en fonction de $z / \delta . H / D=6,25, S / R e=2 \times 10^{-6}$.

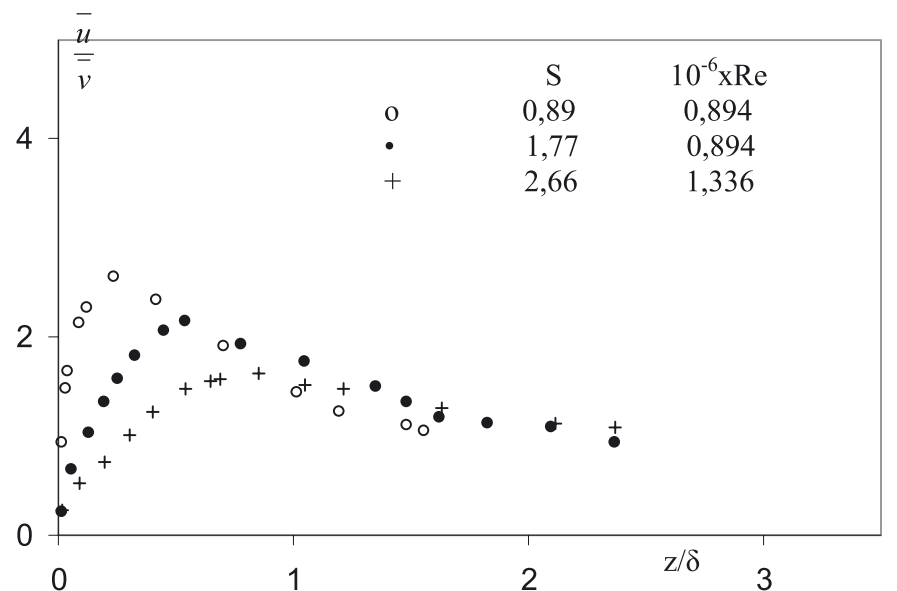

(a)

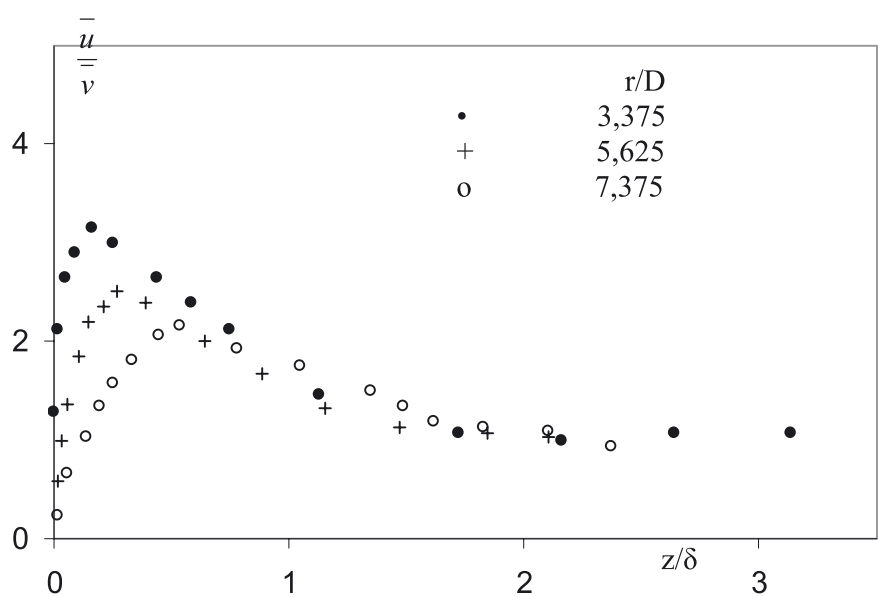

(b)

Fig. 4. (a) Rapport des vitesses radiales aux vitesses tangentielles en fonction de $z / \delta$. $H / D=6,25, r / D=7,375$. (b) Rapport des vitesses radiales aux vitesses tangentielles en fonction de $z / \delta . H / D=6,25, S=1,77, R e=0,894 \times 10^{6}$. 


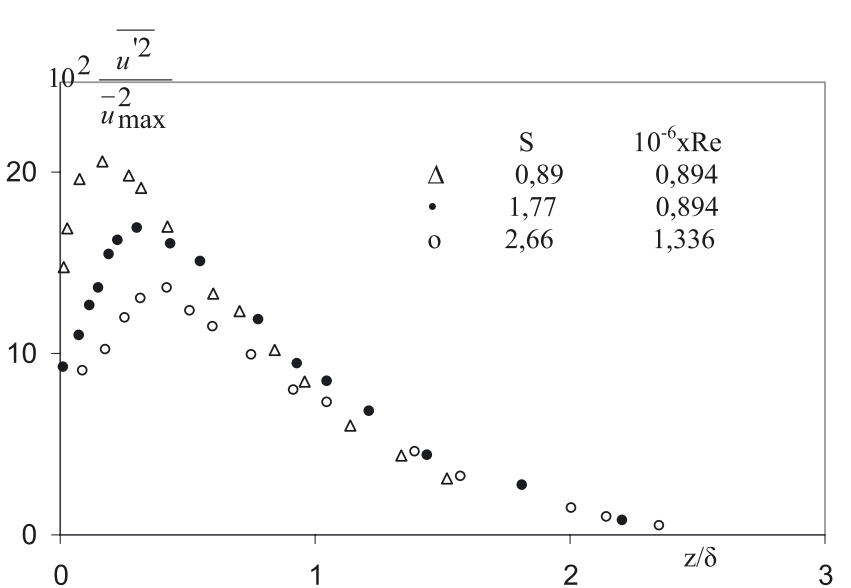

(a)

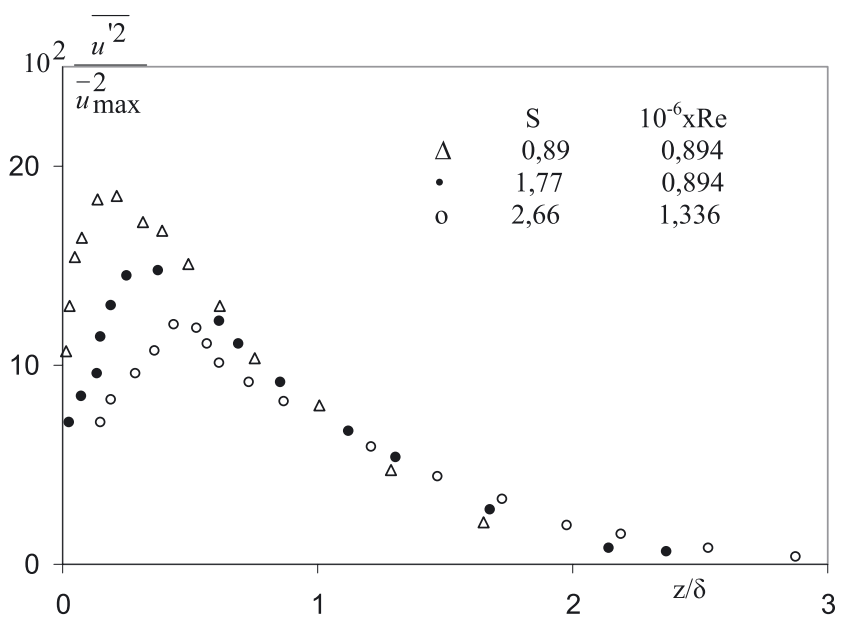

(b)

Fig. 5. (a) Contraintes turbulentes radiales réduites en fonction de $z / \delta . H / D=6,25, r / D=6,375$. (b) Contraintes turbulentes radiales réduites en fonction de $z / \delta$. $H / D=2,5, r / D=6,375$.

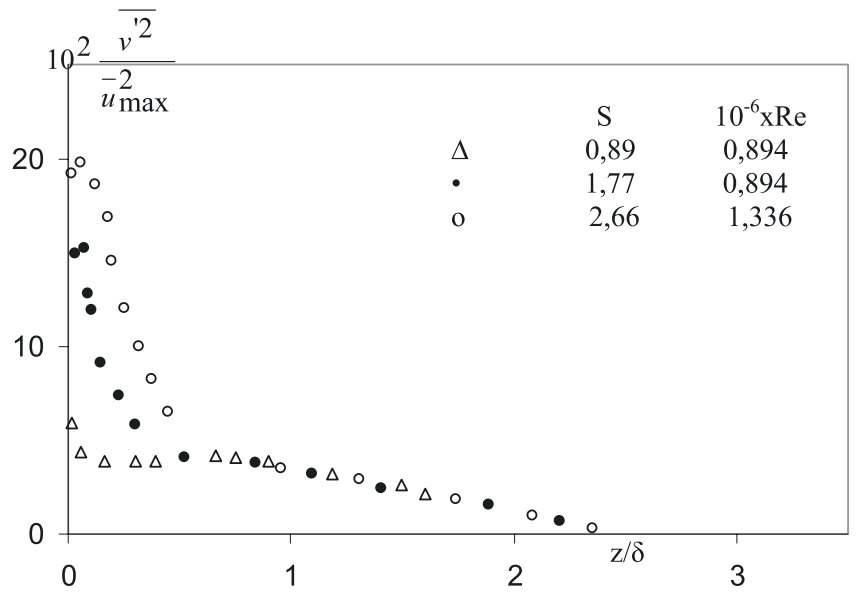

(a)

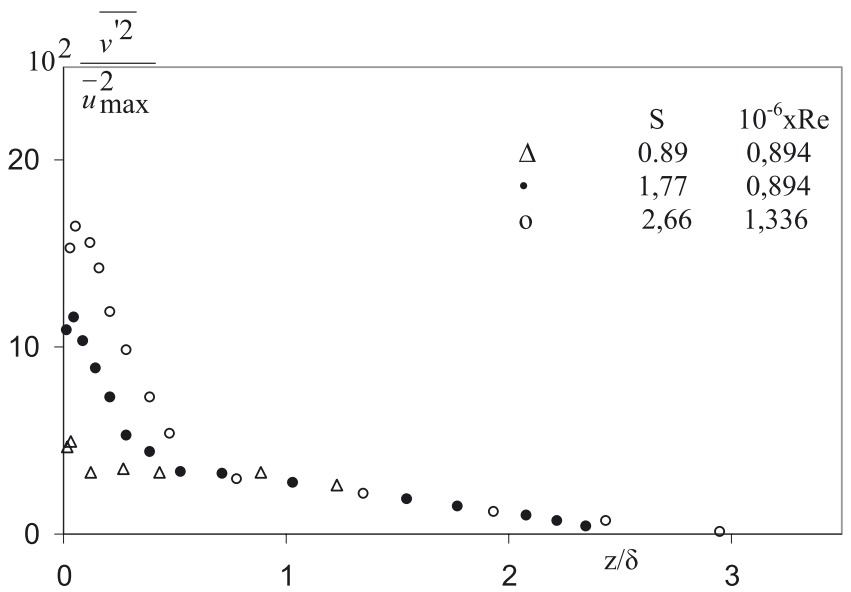

(b)

Fig. 6. (a) Contraintes turbulentes tangentielles réduites en fonction de $z / \delta$. $H / D=6,25, r / D=6,375$. (b) Contraintes turbulentes tangentielles réduites en fonction de $z / \delta$. $H / D=2,5, r / D=6,375$.

La turbulence diminue légèrement dans le sens radial pour $S, R e$ et $H / D$ donnés. Pour de faibles positions radiales ou de faibles valeurs de $S$ et $R e$, on retrouve les résultats du jet purement radial [9]. En revanche, quand $r / D$ ou $S$ sont élevés, les résultats expérimentaux sont proches de ceux d'un écoulement produit par un disque en rotation, sans alimentation radiale. En particulier, le maximum de l'intensité de la turbulence radiale s'éloigne de la surface du disque.

Par ailleurs, lorsque $H / D$ diminue, $\frac{\overline{u^{\prime 2}}}{\overline{u_{\max }^{2}}}$ diminue aussi, $S$, Re et $r / D$ fixés.

\subsubsection{Contrainte turbulente tangentielle}

Les profils de $\frac{\overline{v^{\prime 2}}}{\bar{u}_{\max }^{2}}$ en fonction de $z / \delta$ sont représentés ici par les figures $6 \mathrm{a}$ et b avec $S$ et $R e$ pour paramètres.
Dans la région externe, on obtient un profil unique. En revanche, dans la zone interne $S$ et $R e$ ont beaucoup d'influence, notamment pour les grandes positions radiales : $\frac{\overline{v^{\prime 2}}}{\overline{u_{\max }^{2}}}$ augmente quand $S$ et Re augmentent. L'influence de $H / D$ est faible. Il y a une légère augmentation de $\frac{\overline{v^{\prime 2}}}{\overline{u_{\max }^{2}}}$ lorsqu'on diminue $H / D ; S$, Re et $r / D$ étant fixés.

\subsubsection{Contraintes de cisaillement}

Les figures $7 \mathrm{a}$ à 9 représentent les intensités des contraintes turbulentes de cisaillement sous la forme : $\frac{\bar{u}^{\prime}{ }^{\prime}}{\bar{u}_{\max }^{2}}, \frac{\bar{u}^{\prime}{ }^{\prime}}{\bar{u}_{\max }^{\prime}}, \frac{\bar{v}^{\prime} w^{\prime}}{\bar{u}_{\max }^{2}}$ en fonction de $z / \delta$ pour différentes valeurs de $S$ et de $R e$, avec $H / D$ et $r / D$ fixés .

Ces figures montrent que les profils $\frac{\overline{u^{\prime} v^{\prime}}}{\bar{u}_{\max }^{2}}$ et $\frac{\overline{u^{\prime} w^{\prime}}}{\bar{u}_{\max }^{2}}$ sont peu influencés par la variation de $S$ et de Re dans les deux 


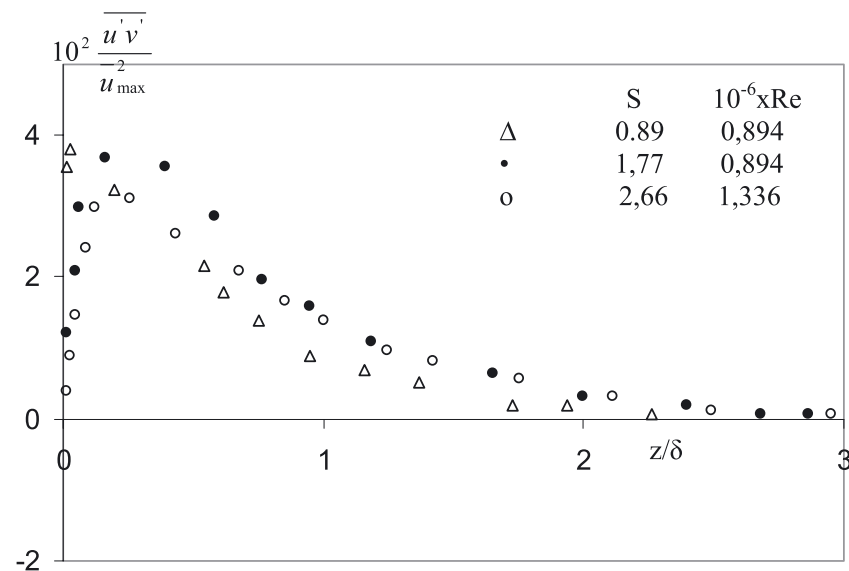

(a)

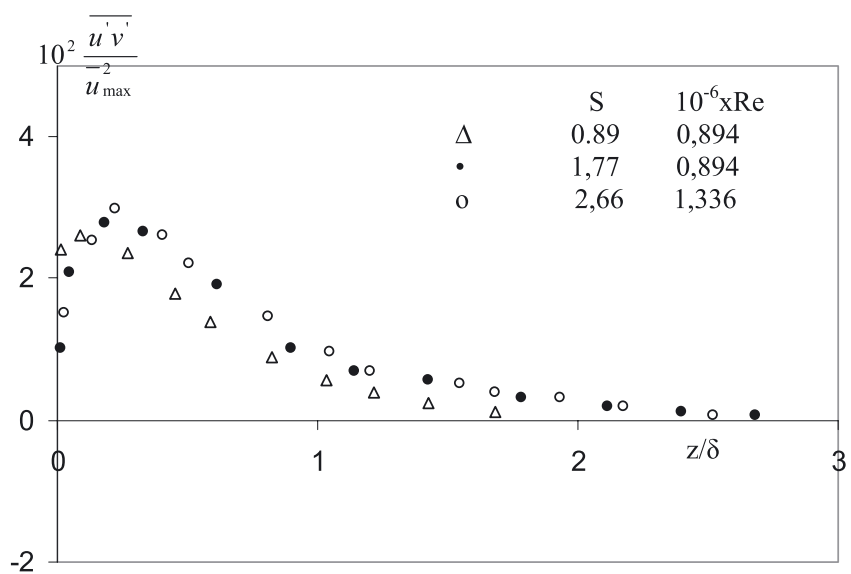

(b)

Fig. 7. (a) Contraintes turbulentes de cisaillement $\overline{u^{\prime} v^{\prime}}$ réduites en fonction de $z / \delta . H / D=6,25, r / D=3,75$. (b) Contraintes turbulentes de cisaillement $\overline{u^{\prime} v^{\prime}}$ réduites en fonction de $z / \delta . H / D=2,5, r / D=3,75$.

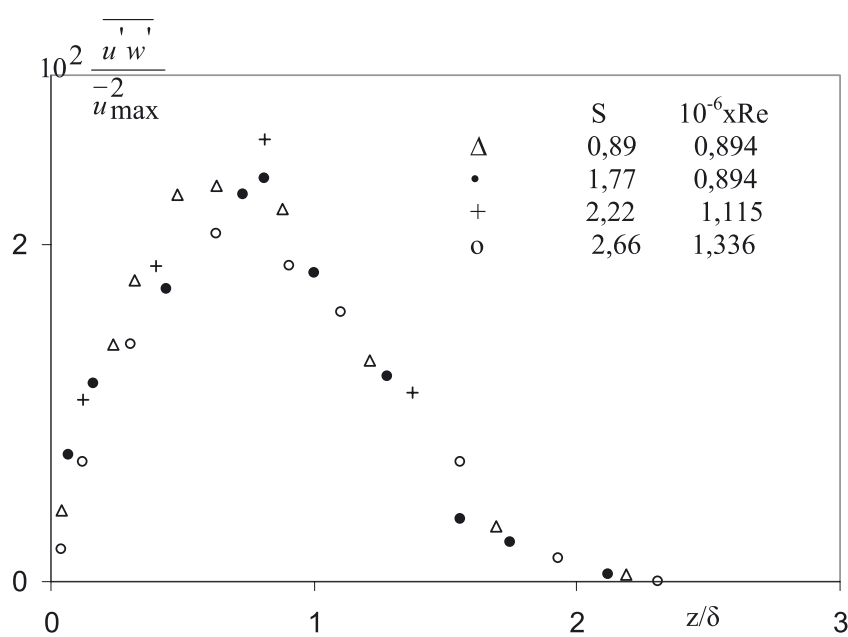

Fig. 8. Contraintes turbulentes de cisaillement $\overline{v^{\prime} w^{\prime}}$ réduites en fonction de $z / \delta$. $H / D=6,25, r / D=3,75$.

régions internes et externes. En ce qui concerne $\frac{\overline{u^{\prime} w^{\prime}}}{\overline{u_{\max }^{2}}}$, il apparaît qu'en dehors du voisinage immédiat de la paroi, la présence du disque n'affecte pas cette contrainte, puisque les résultats obtenus sont presque similaires à ceux correspondant au jet tournant libre dans sa partie supérieure par rapport au disque tournant.

Le paramètre $H / D$ a une faible influence sur $\frac{\overline{u^{\prime} v^{\prime}}}{\bar{u}_{\max }^{2}}$ : à $S$, Re et $r / D$ donnés on constate que $\frac{\overline{u^{\prime} v^{\prime}}}{\bar{u}_{\max }^{2}}$ varie légèrement dans le même sens que $H / D$.

Les profils de $\frac{\overline{v^{\prime} w^{\prime}}}{\bar{u}_{\max }^{2}}$ dépendent d'une part de $\frac{S}{R_{e}}=$ $\frac{\pi D^{2} v}{4 R Q}$, d'autre part de $H / D$ et de $r / D$. $\frac{\overline{v^{\prime} w^{\prime}}}{\bar{u}_{\max }^{2}}$ augmente lorsque $\frac{\pi D^{2} v}{4 R Q}$ diminue pour $H / D$ et $r / D$ fixés, et varie dans le même sens que $H / D$ pour $r / D$ et $\frac{\pi D^{2} v}{4 R Q}$ donnés.

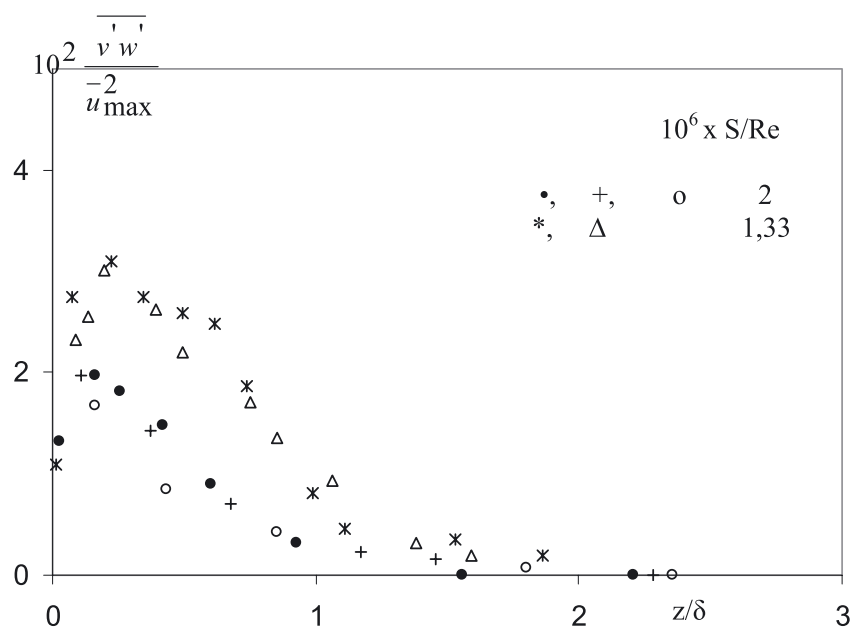

Fig. 9. Contraintes turbulentes de cisaillement $\overline{v^{\prime} w^{\prime}}$ réduites en fonction de $z / \delta . H / D=6,25, r / D=3,75$.

\section{3 Échelles caractéristiques}

\subsection{1 Échelle de vitesse moyenne}

L'échelle caractéristique des vitesses radiales moyennes est la vitesse $\bar{u}_{\max }$, celle des vitesses tangentielles est $2 \pi \mathrm{Nr}$. La figure 10 représente $W_{0} / \bar{u}_{\max }$ en fonction de $r / D$ pour différentes valeurs de $S, R e$, le paramètre $H / D$ étant fixé, cette représentation étant faite dans le but de faire la comparaison avec la courbe théorique du jet purement radial, qui est une droite.

On constate que lorsque $S$ est petit, les résultats expérimentaux concernant $W_{0} / \bar{u}_{\max }$ s'approchent de la droite correspondant au jet purement radial et cela d'autant plus que $r / D$ est faible. Par contre, pour des valeurs plus grandes de $r / D$, lorsque $S$ est grand, les valeurs de $W_{0} / \bar{u}_{\max }$ s'écartent beaucoup plus de cette droite. Le paramètre $H / D$ a une faible influence sur $W_{0} / \bar{u}_{\max }$ à $S$ et Re fixés. 


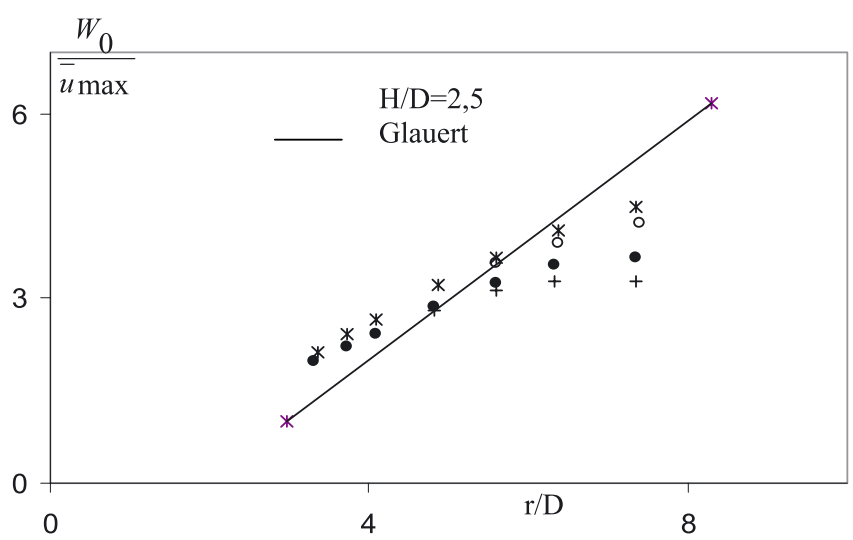

Fig. 10. Vitesse moyenne à la sortie du tube réduite en fonction de $r / D$. Le couple $\left(S, 10^{-6} \times R e\right)$ prend les valeurs suivantes : X $(1,18,0,894), \bullet(1,77,0,894),+(2,22,1,115)$, $\circ(2,66,1,336)$.

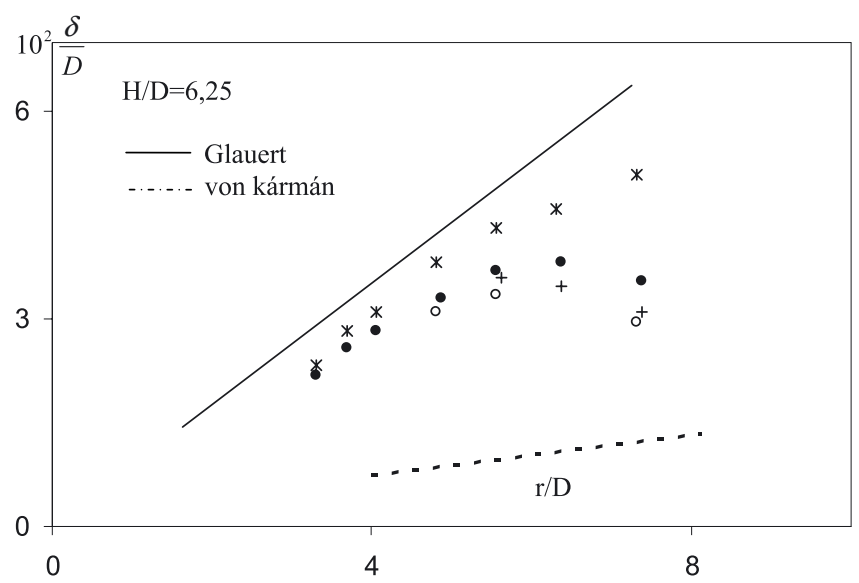

Fig. 11. Épaisseur réduite en fonction de $r / D$. Le couple $\left(S, 10^{-6} \times R e\right)$ prend les valeurs suivantes : X $(1,18,0,894)$, - $(1,77,0,894),+(2,22,1,115)$, 。 $(2,66,1,336)$.

\subsection{2 Échelle de longueur}

La figure 11 représente $\delta / D$ en fonction de $r / D$ avec $S$ et $R e$ comme paramètres et $H / D$ fixé. Les résultats expérimentaux sont situés entre deux droites : celle correspondant au jet purement radial dont la pente est environ 0,087 et celle correspondant à l'écoulement de Von Kármán. Là aussi, on constate que lorsque $S$ diminue, les résultats expérimentaux de $\delta / D$ s'approchent de la courbe correspondant au jet purement radial, d'autant plus que $r / D$ est faible. En revanche, lorsque $S$ augmente les valeurs de $\delta / D$ s'approchent de celles de Von Kármán, notamment pour les grandes positions radiales. Le paramètre $H / D$ n'intervient pas beaucoup, comme on peut le constater sur la même figure.

\section{Validation de l'approximation de couche limite}

Les équations (17) à (19) décrivant l'écoulement dans la zone II sont obtenues à partir des relations (5), (6),
(7) et (8), en négligeant certains termes dans le cadre de l'approximation de couche limite. Dans ce paragraphe, en se servant des résultats expérimentaux obtenus, on va évaluer l'ordre de grandeur des termes négligés afin de justifier cette approximation.

En utilisant (5), on peut écrire l'équation (8) sous la forme suivante :

$$
\begin{aligned}
\frac{\partial}{\partial z}\left(\bar{w}^{2}+\overline{w^{\prime 2}}-\right. & \left.v \frac{\partial \bar{w}}{\partial z}\right)+\frac{1}{\rho} \frac{\partial \bar{p}}{\partial z}+\frac{1}{r} \frac{\partial}{\partial r} \\
\times & {\left[r\left(\overline{u w}+\overline{\left.u^{\prime} w^{\prime}\right)}-v \frac{\partial(r \bar{w})}{\partial r}\right]=0\right.}
\end{aligned}
$$

De l'équation de continuité, on déduit que $w$ est de l'ordre de grandeur $\delta \bar{u}_{\max } / r$.

Le terme $v \frac{\partial \bar{w}}{\partial z}$ représente l'effet de la viscosité. On va le comparer à $\bar{w}^{2}$, en évaluant l'ordre de grandeur du rapport $v \frac{\partial \bar{w}}{\partial z} / \bar{w}^{2}$.

L'ordre de grandeur de $v \frac{\partial \bar{w}}{\partial z} / \bar{w}^{2}$ est $\frac{1}{\frac{\delta}{r} \frac{\bar{u}_{\max }}{v}} \approx$ $\frac{1}{\frac{\delta}{r} \frac{3 \bar{z}_{\max } D}{v} \frac{\delta}{D}} \approx \frac{1}{3 \frac{\delta}{r} \operatorname{Re} \frac{\delta}{D}} \cdot \bar{u}_{\max } \approx 3 \bar{v}_{\max }$ d'après [10]. Le nombre de Reynolds $R e$ est d'ordre $10^{3}$. Les valeurs expérimentales de $\frac{\delta}{r}$ (Figs. 11a et b) sont proches de celles correspondant au jet purement radial pour lequel $\frac{\delta}{r} \cong 0,087$. $\frac{\delta}{D}$ est d'ordre environ 0,3 pour les faibles positions radiales et 0,5 pour les grandes valeurs de $\frac{r}{D}$, ce qui donne $v \frac{\partial \bar{w}}{\partial z} / \bar{w}^{2}$ d'ordre environ $10^{-3}$.

On peut également démontrer que le terme $\frac{v}{r \overline{u w}} \frac{\partial(r \bar{w})}{\partial r}$ est d'ordre $\frac{1}{3 \frac{r}{D} R e} \approx 10^{-4}$, ce qui peut, donc, être négligé. Cela étant, en intégrant (21) de $z$ à l'infini, on obtient :

$$
\frac{\bar{p}-p_{\infty}}{\rho}=\int_{z}^{\infty} \frac{1}{r} \frac{\partial}{\partial r}\left[r\left(\overline{u w}+\overline{u^{\prime} w^{\prime}}\right)\right] \mathrm{d} z+\left(w_{\infty}-\bar{w}^{2}\right)-\overline{w^{\prime 2}}
$$

Généralement, dans les écoulements turbulents de cisaillement, $\overline{w^{\prime 2}}$ est du même ordre de grandeur que $\overline{u^{\prime 2}}$ [19]. D'après les résultats expérimentaux, $\overline{u^{\prime 2}} \cong 0,2 \bar{u}_{\max }^{2}$, $\overline{u^{\prime} w^{\prime}} \cong 0,023 \bar{u}_{\text {max }}^{2}, \overline{u w} \cong 0,087 \bar{u}_{\text {max }}^{2}$ dans le cas de faibles positions radiales, et dans le cas des grandes valeurs de $r / D$ et $/$ ou $S$ et $R e: \overline{u^{\prime 2}} \cong 0,15 u_{\max }^{2}, \overline{u^{\prime} w^{\prime}} \cong 0,023 \bar{u}_{\max }^{2}$, $\overline{u w} \cong 0,02 u_{\max }^{2}$. En admettant $w_{\infty} \cong w(z)$, le terme de pression devient de l'ordre de $-0,21 \bar{u}_{\max }^{2}$ dans le premier cas et de $-0,151 \bar{u}_{\max }^{2}$ dans le second cas.

On peut également écrire l'équation (6) sous la forme suivante, en utilisant l'équation de continuité :

$$
\begin{aligned}
\frac{\partial}{\partial r}\left(\frac{1}{2} \bar{u}^{2}+\overline{u^{\prime 2}}-\frac{\bar{p}-p_{\infty}}{\rho}\right)+\bar{w} \frac{\partial \bar{u}}{\partial z}-\frac{\bar{v}^{2}}{r} \\
=\frac{\partial}{\partial z}\left(v \frac{\partial \bar{u}}{\partial z}-\overline{u^{\prime} w^{\prime}}\right)+\frac{\overline{v^{\prime 2}}-\overline{u^{\prime 2}}}{r}
\end{aligned}
$$

Les mesures effectuées montrent que pour les grandes positions radiales et les grandes valeurs de $S, \overline{v^{\prime 2}}$ est à peu près du même ordre que $\overline{u^{\prime 2}}$. On en conclut que le dernier terme de l'équation précédente peut être négligé. En revanche, pour les faibles positions radiales, $\overline{v^{\prime 2}}$ est de 
l'ordre de $0,5 \overline{u^{\prime 2}}$. Comme par ailleurs $\overline{u^{\prime 2}}$ est de l'ordre de $0,2 \bar{u}_{\max }^{2}$, on déduit que $\frac{\overline{v^{\prime 2}}-\overline{u^{\prime 2}}}{r}$ est d'ordre de $-0,1 \frac{\bar{u}_{\max }^{2}}{r}$. En négligeant $\frac{\overline{v^{\prime 2}}-\overline{u^{\prime 2}}}{r}$ et $\overline{u^{\prime 2}}+\left(\bar{p}-p_{\infty}\right) / \rho$, on commet une erreur ne dépassant pas $1 \%$ dans le premier cas et $10 \%$ dans le second cas.

De la même manière l'équation (7) peut s'écrire :

$$
\frac{1}{r^{2}} \frac{\partial}{\partial r}\left(r^{2}\left(\overline{u v}+\overline{u^{\prime} v^{\prime}}\right)\right)+\frac{\partial(\overline{v w})}{\partial z}=\frac{\partial}{\partial z}\left(v \frac{\partial \bar{v}}{\partial z}-\overline{v^{\prime} w^{\prime}}\right)
$$

$\overline{u^{\prime} v^{\prime}}$ est d'ordre de $0,04 \bar{u}_{\max }^{2}$ environ. Le rapport $\overline{u^{\prime} v^{\prime}} / u v$ est donc d'ordre 0,04 $\bar{u}_{\max } / \bar{v}_{\max }$. Or, $\bar{u}_{\max }$ est toujours inférieure à $\bar{v}_{\max }$ (on a $\bar{u}_{\max } \cong \bar{v}_{\max }$ pour $r$ petit). Par conséquent on a $\overline{u^{\prime} v^{\prime}} \cong 0,04 \overline{u v}$. Ainsi en négligeant $\overline{u^{\prime} v^{\prime}} / \overline{u v}$, on commet une erreur inférieure ou égale à $4 \%$.

De l'analyse des ordres de grandeurs des différents termes des équations de la dynamique, on peut conclure que les approximations de couche limite appliquées à notre problème entraînent des erreurs, notamment pour la projection radiale. Pour obtenir une précision meilleure, les équations (6) et (7) doivent être remplacées par :

$$
\begin{array}{r}
\bar{u} \frac{\partial \bar{u}}{\partial r}+\bar{w} \frac{\partial \bar{u}}{\partial z}-\frac{\bar{v}^{2}+\overline{v^{\prime 2}}}{r}=\frac{\partial}{\partial z}\left(\frac{\tau_{r z}}{\rho}-\overline{u^{\prime} w^{\prime}}\right) \\
-\frac{1}{r} \frac{\partial r \overline{u^{\prime 2}}}{\partial r}+\frac{\partial \overline{w^{\prime 2}}}{\partial r} \\
\bar{u} \frac{\partial \bar{v}}{\partial r}+\bar{w} \frac{\partial \bar{v}}{\partial z}+\frac{\overline{u v}}{r}=\frac{\partial}{\partial z}\left(\frac{\tau_{\theta z}}{\rho}-\overline{v^{\prime} w^{\prime}}\right) \\
-\frac{1}{r^{2}} \frac{\partial}{\partial r}\left(r^{2} \overline{\left.u^{\prime} v^{\prime}\right)}\right.
\end{array}
$$

\section{Conclusion}

L'étude expérimentale décrite dans cet article est consacrée au domaine du jet développé, créé sur un disque en rotation par l'impact d'un jet rond, coaxial. Les mesures ont été faites par anémométrie à fil chaud. Elles fournissent les vitesses moyennes radiales $\bar{u}$ et tangentielles $\bar{v}$, ainsi que les contraintes de Reynolds : $\overline{u^{\prime 2}}, \overline{v^{\prime 2}}$, $\overline{u^{\prime} v^{\prime}}, \overline{u^{\prime} w^{\prime}}$ et $\overline{v^{\prime} w^{\prime}}$.

On distingue usuellement deux zones, l'une proche de la paroi qui est sous influence directe de celle-ci, et une autre, éloignée de la paroi et ayant les caractéristiques de la turbulence libre. L'analyse dimensionnelle du problème a permis de dégager trois paramètres sans dimensions caractéristiques : un nombre de Reynolds Re, un nombre de rotation $S$ et le rapport entre les longueurs, $H$ distance du jet au disque et $D$, diamètre du jet axial. Leur influence sur les paramètres de l'écoulement est beaucoup plus importante dans la région interne, proche de la surface du disque, notamment sur les contraintes turbulentes. Ailleurs, on observe presque une bonne similitude. Concernant $\frac{\bar{u}}{\bar{u}_{\max }}$, on a presque une bonne similitude aussi bien dans la zone proche que loin de la paroi du disque, cela est d'autant plus vrai que les positions radiales sont faibles.

Par ailleurs, $\frac{\bar{v}}{\bar{v}_{\max }}$ ne dépend pas de la rotation du disque.

Les mesures des contraintes de Reynolds ont révélé l'anisotropie du champ turbulent. Ces contraintes calculées par l'utilisation des relations suivantes : $\rho \overline{u^{\prime} v^{\prime}}=$ $\mu_{t}\left(\frac{\partial \bar{v}}{\partial r}-\frac{v}{r}\right), \rho \overline{u^{\prime} w^{\prime}}=\mu_{t} \frac{\partial \bar{u}}{\partial z}, \rho \overline{v^{\prime} w^{\prime}}=\mu_{t} \frac{\partial \bar{v}}{\partial z} ; \mu_{t}$ viscosité turbulente ne sont pas en concordance avec les résultats de nos essais [10], comme pour le jet purement radial [9].

Habituellement, on utilise pour tous les écoulements de cisaillement les approximations de la couche limite. Nos expériences ont permis d'évaluer les ordres de grandeurs des termes négligés lorsque cette approximation est adoptée. On constate que les équations simplifiées de la dynamique sont légèrement erronées, surtout dans le sens radial, à cause du fait qu'on a négligé les termes des contraintes turbulentes.

L'étude présentée dans cet article est fondamentale. Elle peut, néanmoins, trouver des applications dans des domaines tels l'électrochimie avec par exemple les électrodes à disque tournant, les aéroglisseurs, coussin d'air, mais aussi en turbomachines.

\section{Références}

[1] P. Cooper, Turbulent boundary layer on a rotating disk calculated with an effective viscosity, AIAA J. 9 (1971) $255-261$

[2] N. Leprovost, L. Marié, B. Dubrulle, A stochastic model of torques in von Kármán swirling flow, Eur. Phys. J. B 39 (2004) 121-129

[3] M. Lygren, H. Andersan, Large eddy simulations of the turbulent flow between a rotating and a stationnary disk, Z. Angew. Maths. Phys. 55 (2004) 268-281

[4] H. Xu, S.-J. Liao, Series solutions of unsteady MHD flows above a rotating disk, MecCanica 41 (2006) 599-609

[5] A.M. Jawarneh, G.H. Vatistas, Reynolds Stress Model in the prediction of confined turbulent swirling flows, J. Fluid Eng. 128 (2006) 1377-1382

[6] S. Poncet, Écoulements de type rotor stator soumis à un flux axial : de Batchelor à Stewartson, Thèse de doctorat, Université de Provence Aix-Marseille, France, 2005

[7] M. Miklavcic, C.Y. Wang, The flow due a rough disk, Z. Angew. Math. Phys. (Birkauser Verlag) 55 (2004) 235246

[8] M.B. Glauert, The wall jet, J. Fluid Mech. 1 (1956) 625643

[9] M. Poreh, Y.G. Tsuel, J.E. Cermak, Investigation of a turbulent radial wall jet, J. Appl. Mech. (1967) 457-463 
[10] K. Boussafeur, Étude expérimentale de l'écoulement turbulent pariétal produit par l'impact d'un jet frappant normalement un disque coaxial en rotation, Thèse Docteur Ingénieur, université de Lille, France, 1989

[11] S. Poncet, M.P Chauve, Instabilité d'une couche de cisaillement au dessus d'un disque en rotation, $18^{\mathrm{e}}$ congrès français de mécanique, France, 2007

[12] J.F. Brady, L. Durlofsky, On rotating disk flow, J. Fluid Mech. 175 (1987) 363-394

[13] R.N. Parthasarathy, Analysis of turbulent boundary layer on a rotating disk, Microsystem technologies (SpringerVerlag) 8 (2002) 278-281
[14] J.J. Healey, Instabilities of flows due to rotating disks : preface, J. Eng. Math. (Springer Science) 57 (2007) 199204

[15] T. Cebeci, D.E. Abott, Boundary layer on a rotating disk, AIAA 13 (1975) 829-832

[16] T.S. Cham, M.R. Head, Turbulent boundary layer flow on a rotating disk, J. Fluid Mech. 37 (1969) 129-147

[17] P. Bakke, An experimental investigation of a wall jet, J. Fluid Mech. 2 (1957) 467-472

[18] F.F. Erian, Y.H. Tong, Turbulent flow due to a rotating disk, Phys. Fluids 14 (1971) 2588-2591

[19] J.O. Hinze, Turbulence, Mc Graw-Hill, 1975 